\title{
Vetores de tripanosomatídeos (Kinetoplastida: Trypanosomatidae) em Taba- tinga, Amazonas, Brasil
}

\author{
Fabiane Veloso Soaresab*, Rui Alves de Freitasa, Luanda de Paula Figueiraa, Antonia Maria Ramos Franco ${ }^{\mathrm{a}}$ \\ a Laboratório de Leishmaniose e Doença de Chagas, Instituto Nacional de Pesquisas da Amazônia, Manaus, 69060-001, \\ Brasil, *afranco@inpa.gov.br \\ b Programa de Pós-Graduação Mestrado em Ciências da Saúde, Universidade Federal do Amazonas, Manaus, 69025-050, Brasil.
}

Recebido: 14 janeiro 2017 / Aceito: 24 fevereiro 2017 / Publicado online: 23 maio 2017

\begin{abstract}
Resumo
Os flebotomíneos transmitem doenças a humanos e animais, como as arboviroses e bartonelose, além das leishmanioses. O objetivo desta pesquisa foi identificar possíveis espécies de flebotomíneos vetores da doença nessa região no município de Tabatinga. Flebotomíneos foram capturados com armadilhas luminosas e em coletas manuais por aspiração em bases das árvores. Nos meses de agosto dos anos 2010 e 2011, foram capturados 4.542 flebotomíneos ( $0: 71,9 \%$ e $\bigcirc: 28,1 \%$ ) vetores ou suspeitos de transmitirem tripanosomatídeos, pertencentes a 13 espécies. A espécie mais abundante foi Lutzomyia umbratilis com 78,7\%. Um total de 576 (74,0\%) flebotomíneos do gênero Lutzomyia foram dissecados, sendo observada infecção natural por tripanosomatídeos em 44 fêmeas. Observou-se no trato digestório dos flebotomíneos a presença de sangue em decomposição em 86,3\%; sendo que no que se refere aos ovários, 59,0\% mostravam-se em desenvolvimento. A análise isoenzimática de dez isolados, demonstrou perfis similares a Leishmania colombiensis e Endotrypanum sp., no entanto, o perfil do locus Malato Desidrogenase (MDH) demonstrou maior similaridade para o gênero Endotrypanum. Esta é a primeira investigação da fauna entomologia e tentativa de isolamento de tripanosomatídeos no município de Tabatinga, área de tríplice fronteira com o Brasil.
\end{abstract}

Palavras-chave: Amazonas, Endotrypanum, flebotomíneos, Lutzomyia.

\section{Vectors of Trypanosomatids (Kinetoplastida: Trypanosomatidae) in Tabatinga, Amazonas, Brazil}

\begin{abstract}
The sand flies transmit diseases to humans and animals, such as arboviruses and bartonellosis, besides the leishmaniasis. The objective of this research was identified potential vectors of this disease in the municipality of Tabatinga. Sand flies were captured with miniature light trap and manual collection by aspiration at the bases of trees. In the months of august 2010 and 2011 , were captured 4,542 sand flies $(\delta: 71.9 \%$ and $+: 28.1 \%)$ vectors or suspected of trypanosomatids transmission, belonging to 13 species. The most abundant species was Lutzomyia umbratilis with 78,7\%. A total of $576(74.0 \%)$ sand flies of the genus Lutzomyia were dissected and natural infection by trypanosomatids was observed in 44 females. Blood in decomposition was detected at the digestive tract in $86,3 \%$ insects; being $59.0 \%$ of the ovaries in developing. The isoenzymatic analysis of ten isolates showed similar profiles to Leishmania colombiensis and Endotrypanum sp., however, the profile of the locus Malate Dehydrogenase (MDH) showed major similarity to the genus Endotrypanum. This is the first investigation of the fauna entomology and attempt of trypanosomatids isolation in the municipality of Tabatinga, area of triple border with Brazil.
\end{abstract}

Keywords: Amazonas, Endotrypanum, sand fly, Lutzomyia.

\section{Introdução}

Flebotomíneos (Diptera: Psychodidae) são insetos de importância médica e veterinária, sendo responsáveis pela transmissão de várias doenças, tais como: bartonelose, arboviroses e leishmanioses visceral e tegumentar (Sherlock, 2003). Na região neotropical, eles são agrupados em três gêneros diferentes: Brumptomyia (França e Parrot, 1921), Warileya (Hertig, 1948) e Lutzomyia (França, 1924). Apenas o ultimo gênero inclui espécies de importância médica para a transmissão da leishmaniose no Novo Mundo.

A Leishmaniose Tegumentar Americana (LTA) é uma zoonose de ampla distribuição mundial. No Brasil são reconhecidas atualmente pelo menos sete espécies dermotrópicas (Leishmania (Viannia) guyanensis (Floch, H. 1954), L. (V.) naiffi (Lainson \& Shaw, 1989), L. (V.) braziliensis (Vianna, G. 1911), L. (V.) lainsoni (Silveira, Shaw, Braga \& Ishikawa, 1987), L. (V.) shawi (Lainson, Braga \& de Souza, 1989), L. (V.) lindenbergi (Silveira, Ishikawa \& de Souza, 2002) e L. (Leish- 
mania) amazonensis (Lainson \& Shaw, 1972 (inclui garnhami Scorza et al., 1979) de interesse médico na Amazônia Brasileira (Silveira et al., 2008; Brasil, 2017). Nas Américas (incluindo o Brasil), a espécie $L$. (L.) chagasi é o agente causador da Leishmaniose Visceral Americana (LVA) ou Calazar neo-tropical (Gontijo e Melo, 2004; Brasil, 2017).

Em toda região amazônica, os flebotomíneos apresentam uma elevada riqueza de espécies (Young e Duncan, 1994) com elevados índices de diversidade local em florestas de terra firme (Barrett et al., 1996; Chagas et al., 2016).

Estudos têm implicado os flebotomíneos como hospedeiros e transmissores de inúmeros micro-organismos (Shaw et al., 2003) tornando sua identificação de fundamental importância para se avaliar susceptibilidade, sensibilidade a substâncias tóxicas e eventual importância epidemiológica (Marcondes et al., 2001; Pirajá e Lucheis, 2014).

O conhecimento da fauna dos flebotomíneos vetores em regiões mais distantes de Manaus (Amazonas), como o município de Tabatinga, ainda é escasso. Para os poucos casos de LTA ocorridos nesse município, até o momento, não se tem conhecimento dos possíveis vetores. Deste modo, a identificação das áreas de risco à transmissão das leishmanioses faz-se necessária para a atuação das vigilâncias ambiental e epidemiológica na prevenção desta doença. Esta pesquisa teve como objetivo identificar possíveis espécies de flebotomíneos vetores da doença nessa região.

\section{Material e Métodos}

\section{Área de estudo}

A pesquisa foi realizada no município de Tabatinga $\left(4^{\circ} 15^{\prime}\right.$ 9" S, 69 56' 17" O), Estado do Amazonas, Brasil, área de fronteira com a Colômbia e Peru, distante $1.105 \mathrm{~km}$ de Manaus, localizado à margem esquerda do Rio Solimões. O município possui $3.239 \mathrm{~km}^{2}$, altitude $60 \mathrm{~m}$. Sua população é de 62.346 habitantes (IBGE, 2016) e o clima é equatorial, com temperatura média de $25^{\circ} \mathrm{C}$. Apresenta uma estação chuvosa entre os meses de novembro e abril, e uma estação seca entre maio e outubro.

\section{Captura de Flebotomíneos}

As coletas foram realizadas ao longo da Estrada do Instituto Nacional de Colonização e Reforma Agrária - INCRA, Geodésica II, entre os km 4 e 8 . Nestas áreas pode-se observar a existência de assentamentos com plantações de banana e mandioca para subsistência. O critério de seleção dos locais de captura foi baseado na ocorrência de casos humanos de LTA.

O levantamento da fauna flebotomínica foi realizado durante 15 dias nos meses de agosto nos anos de 2010 e 2011, totalizando 30 dias. Os flebotomíneos foram coletados utilizando-se armadilhas luminosas do tipo CDC modelo 512 (CDC "miniature" - Hausherr' Machine Works, New Jersey, EUA) instaladas em áreas de mata de terra firme e no peridomicílio a um metro do solo, entre as 18:00h e 6:00h do dia seguinte. Também foram feitas coletas por aspiração em base de árvores com armadilha do tipo CDC modificada no momento da retirada das armadilhas luminosas entre $8 \mathrm{~h}$ e $8 \mathrm{~h} 30 \mathrm{~m}$. Posteriormente, os insetos foram triados, condicionados em etanol e identificados segundo a chave taxonômica de Young e Duncan
(1994). As fêmeas foram dissecadas para observação e isolamento de flagelados.

\section{Dissecção de flebotomíneos e infecção natural por flagelados}

As fêmeas retiradas da gaiola de náilon foram lavadas em solução detergente a $2 \%$ e depois transferidas para solução de salina estéril $(0,9 \%)$; foram retirados os dois últimos tergitos abdominais e logo depois o aparelho digestivo; o conteúdo do tubo digestivo foi analisado em microscópio ótico para a ocorrência e localização dos protozoários; quando positivo, era feito aspirado e semeado em meio de cultura bifásico Novy e MacNeal; Nicolle (NNN) [Novy e MacNeal, 1904; Nicolle, 1908]. As fases das mudanças observadas nos ovários estão de acordo com Christophers e Mer (Forattini, 1973), onde 1 = sem visualização de oócitos; 2 = oócitos nas fases II e III de desenvolvimento e 3 = oócitos nas fases IV e V de desenvolvimento. Os parasitos isolados foram mantidos em meio de cultivo bifásico $\mathrm{NNN}$, acrescidos de fase liquida de Schneider (Sigma) (Hendricks et al., 1978) completo suplementado por $10 \%$ de soro fetal bovino inativado (SFBi/Cultilab) e repicados em intervalos semanais. Os isolados foram amplificados e criopreservados em nitrogênio líquido, sendo depositados no criobanco de parasitos do Instituto Nacional de Pesquisas da Amazônia (INPA) para a posterior identificação dos flagelados.

Isolados em cultivo foram amplificados para análise de eletroforese de enzimas em gel de agarose (Figueira et al., 2008). A taxa de infecção natural (TIN) para tripanosomatídeos foi obtida pelo cálculo percentual da razão entre o número de fêmeas infectadas e o número de fêmeas dissecadas por espécie (Freitas et al., 2002).

\section{Resultados e Discussão}

Foram coletados 4.589 flebotomíneos, abrangendo 13 espécies e, onde sete delas $(53,8 \%)$ têm hábito de picar o homem, principalmente as espécies dos subgêneros Nyssomyia e Psychodopygus (Young \& Arias, 1992). 71,7\% (3.291) dos flebotomíneos era macho e 28,3\% (1.298) fêmeas, distribuídos no gênero Lutzomyia, incriminadas na literatura como vetores comprovados ou suspeitos na transmissão de leishmanias ao homem, bem como outros tripanosomatídeos, nas diferentes regiões do Brasil e do Mundo. Dentre essas espécies, a mais representativa foi a L. umbratilis (Ward RD, Fraiha H. 1977), perfazendo $77,9 \%$ dos espécimes capturados. Seguem na ordem de abundância L. dendrophyla (Mangabeira, 1942), (10,4\%), L. davisi (Root F. M. 1934) (3,4\%), L. shannoni (Dyar, 1929) (2,2\%), L. yuilli (Young \& Porter, 1972) (1,6\%). Outras espécies menos frequentes perfizeram $4,5 \%$ da amostra total (Tabela 1).

Todos os representantes das espécies foram encontrados em área silvestre. Somente sete indivíduos foram capturados no peridomicílio (chiqueiro e galinheiro), distribuídos entre as espécies L. umbratilis (02 flebotomíneos), L. ayrozai (Barretto M. P., Coutinho J. O., 1940) (01), L. yuilli (01), L. furcata (Mangabeira Fo O., 1941) (02) e L. walkeri (Newstead R., 1914) (01). Foram coletados 823 (18,0\%) flebotomíneos em armadilhas luminosas tipo CDC e $3.766(82,0 \%)$ por aspiração em base de árvores. 
Tabela 1. Flebotomíneos incriminados como transmissores de tripanosomatídeos, capturados em área silvestre e peridomicílio* no município de Tabatinga, Amazonas, Brasil (períodos de agosto de 2010 e agosto de 2011).

\begin{tabular}{|c|c|c|c|c|c|c|c|c|c|}
\hline \multirow{4}{*}{ Espécie } & \multicolumn{6}{|c|}{ Método de coleta } & \multirow{3}{*}{\multicolumn{2}{|c|}{ Total }} & \multirow{4}{*}{$\begin{array}{l}\text { Total } \\
\text { Geral }\end{array}$} \\
\hline & \multicolumn{4}{|c|}{ Silvestre } & \multirow{2}{*}{\multicolumn{2}{|c|}{$\begin{array}{c}\text { Peridomicílio } \\
\text { CDC }\end{array}$}} & & & \\
\hline & \multicolumn{2}{|c|}{$\mathrm{CDC}$} & \multicolumn{2}{|c|}{ BA } & & & & & \\
\hline & $\pi$ & q & $\hat{0}$ & 오 & $\hat{0}$ & o & 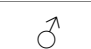 & o & \\
\hline Lutzomyia umbratilis* & 189 & 173 & 2422 & 791 & - & 2 & 2611 & 966 & 3577 \\
\hline L. dendrophyla* & 56 & 7 & 365 & 50 & - & - & 421 & 57 & 478 \\
\hline L. davisi & 68 & 88 & - & 1 & - & - & 68 & 89 & 157 \\
\hline L. shannoni & 9 & - & 84 & 9 & - & - & 93 & 9 & 102 \\
\hline L. yuilli & - & 73 & - & - & - & 1 & - & 74 & 74 \\
\hline L. sordelli & 36 & 22 & - & - & - & - & 36 & 22 & 58 \\
\hline L. tuberculata* & - & 27 & 4 & 6 & - & - & 4 & 33 & 37 \\
\hline L. ayrozai & 19 & 12 & - & - & - & 1 & 19 & 13 & 32 \\
\hline L. antunesi & - & 2 & 21 & 7 & - & - & 21 & 9 & 30 \\
\hline L. furcata & 2 & 15 & 5 & - & 2 & - & 9 & 15 & 24 \\
\hline L. walkeri & 6 & 6 & - & - & - & 1 & 6 & 7 & 13 \\
\hline L. paraensis & 2 & 3 & - & - & - & - & 2 & 3 & 5 \\
\hline L. amazonensis & - & 1 & 1 & - & - & - & 1 & 1 & 2 \\
\hline Total & 387 & 429 & 2902 & 864 & 2 & 5 & 3291 & 1298 & 4589 \\
\hline
\end{tabular}

CDC: Armadilha luminosa; BA: Base de árvore; *Coletas apenas no ano de 2010; $\hat{o}$ - sexo masculino; + - sexo feminino.

A maioria das espécies foi comum tanto em armadilha luminosa tipo CDC quanto em aspiração em base de árvore. As espécies L. dendrophyla, L. antunesi (Coutinho J. O., 1939) e L. shannoni foram pouco atraídas por armadilhas de luz similar ao estudo de Nery et al. (2010). Isso talvez seja devido a predominância dessas espécies nas copas das árvores durante o período noturno (Dias-Lima et al., 2002). Difere desse resultado, os resultados publicados por Barrett et al. (1996) em TeféAmazonas, onde as espécies acima citadas foram observadas em menor quantidade nas coletas em base de árvore.

Os resultados obtidos são semelhantes aos obtidos por pesquisas realizadas em outros municípios do Amazonas como em Itacoatiara (Pessoa et al., 2007), Coari (Gomes et al., 2009) e Rio Preto da Eva (Franco et al., 2010) nas quais L. umbratilis é a espécie mais abundante. Diferem desses resultados os observados por Fé et al. (1998) em São Gabriel da Cachoeira e por Silva et al. (2007) em Manacapuru, ambos no Amazonas. Castellón et al. (1994) reportaram em sua pesquisa realizada na estrada Manaus - Humaitá, que em geral dentre as espécies coletadas, a mais comum foi $L$. antunesi.

A segunda espécie mais abundante em nosso estudo foi $L$. dendrophyla. Essa espécie já foi encontrada com infecção por Trypanosoma sp. no Pará (Ryan et al., 1987) e no Amapá seus flagelados foram identificados por eletroforese de isoenzimas como sendo $L$. (V.) guyanensis (Franco, dados não publicados), mas Freitas et al. (2002) não sugerem que $L$. dendrophyla seja um hospedeiro natural deste parasita. Não há muitos registros na literatura sobre infecção natural nessa espécie.

Uma espécie que chama bastante atenção, apesar de pouco representada aqui é $L$. yuilli, com sua reconhecida importância médica na transmissão de Leishmania para humanos na Colômbia, tendo sido encontrada naturalmente infectada com L. (V.) panamensis (Santamaría et al., 2006), indicando participação ativa na transmissão dessa espécie, que causa mais de 50\% dos casos de LTA no país (Vergara et al., 2008). Em 1998, Ferro e Morales também encontraram essa espécie infectada com flagelados não identificados na cidade de Letícia - Colômbia, fronteira com Tabatinga-AM, Brasil. Além da Colômbia, sua distribuição é conhecida para o Brasil, Bolívia (Vergara et al., 2008), Equador, Peru e Venezuela (Aguiar et al., 2003).

Quanto a infecção natural por tripanosomatídeos em flebotomíneos, foram dissecadas $576(74,3 \%)$ fêmeas. As espécies, total de espécimes e percentual de abundância em relação ao total de espécimes coletadas, foram: L. umbratilis $(462 / 80,2 \%)$, L. dendrophyla $(42 / 7,2 \%)$, L. davisi $(09 / 1,5 \%)$, L. shannoni (07/1,2\%), L. yuilli (08/1,3\%), L. sordelli (Shannon R. C., Del Ponte E., 1927), (04/0,6\%), L. tuberculata (20/3,4\%), L. ayrozai $(02 / 0,3 \%), L$ antunesi $(07 / 1,2 \%)$, L. furcata (09/1,5\%), L. walkeri $(02 / 0,3 \%)$, L. paraensis $(03 / 0,5 \%)$ e $L$. amazonensis $(01 / 0,1 \%)$, sendo observada infecção natural por tripanosomatídeos em 44 (7,6\%) espécimes de L. umbratilis $(34 / 77,2 \%)$, L. dendrophyla $(9 / 20,4 \%)$ e L. tuberculata $(1 / 2,4 \%)$.

O conteúdo estomacal de $86,3 \%$ das fêmeas positivas para infecção apresentou sangue em decomposição, 9,2\% sangue fresco e 4,5\% com ausência de sangue. Quanto às condições dos ovários, 59\% apresentou ovos pequenos, seguido de 31,9\% ovos em desenvolvimento e $9,1 \%$ ovos grandes. Variada carga parasitária foi observada no trato digestório dos insetos dissecados. A maioria $(93,2 \%)$ apresentou de um a cinco flagelados por campo (+), seguido de $4,5 \%$ de seis a 20 flagelados $(++)$ e $2,3 \%$ de 21 a 40 flagelados por campo (+++).

A taxa de infecção natural encontrada em $L$. umbratilis foi de $7,3 \%, L$. dendrophyla de $21,4 \%$ e $L$. tuberculata de $5 \%$. Das 44 amostras positivas e cultivadas em meio de cultivo bifásico NNN, apenas 10 cresceram em cultivo, sendo expandidas em meio líquido Schneider completo. Dentre essas 10 amostras, oito são L. umbratilis (sete coletadas em BA e uma em CDC) e as outras duas são L. dendrophyla (coletadas em BA). As 34 restantes ou sofreram contaminação por micro-organismos e/ ou não ocorreu desenvolvimento parasitário.

A infecção natural é um dos critérios mais importantes para incriminar uma espécie de Lutzomyia como vetor de 
Leishmania (Killick-Kendrick, 1990). No entanto, é de extrema importância que não exista a presença de sangue fresco ou em processo de digestão para que a mesma seja indicada como transmissora.

Em estudo na área de Salobo, na Serra dos Carajás, Pará (Souza et al, 2010) 11.259 (73,5\%) flebotomíneos foram dissecados e examinados para pesquisa de flagelados do gênero Leishmania, sendo encontradas 19 infecções naturais, destes oito foram isolados e em dois exemplares de L. davisi revelou a presença de L. (V.) braziliensis. Neitzke et al. (2008) no Paraná, analisaram 2.487 exemplares e verificaram uma fêmea com flagelados, ou seja, infecção de $0,04 \%$.

Nery et al. (2010), estudando L. umbratilis num fragmento florestal urbano da cidade de Manaus encontraram uma taxa de 8.6\%. Lainson et al. (1981), encontraram no norte do Pará uma taxa de infecção de 1,7\%. Pinheiro (2008) e Gomes et al. (2010) estudando leishmaniose em área de treinamento militar no Amazonas, constataram uma taxa de infecção de $1,66 \%$ e $1,96 \%$, respectivamente. Franco et al. (2010) analisando a fauna de flebotomíneos em área de assentamento em Rio Preto da Eva (AM) observaram uma taxa de 3,89\% para esta espécie. Ready et al. (1985) realizando um estudo piloto de controle de L. umbratilis na área periurbana da cidade de Manaus (bairro de São José) registraram uma taxa de infecção por L. (V.) guyanensis de 7\% e Freitas et al. (2002) em investigações no Amapá assinalaram uma taxa de infecção nestes insetos de 26,5\%. Em relação a análise isoenzimática, foram observadas formas promastigotas na dissecção dos 44 flebotomíneos e em cultivo nas dez amostras isoladas. O resultado da análise bioquímica (eletroforese de isoenzimas em gel de agarose) demonstra similaridade dos perfis isoenzimáticos das amostras, quando comparados com perfis de zimodemas de Endotrypanum sp. (Franco et al., 1996).

Os 10 isolados não desenvolveram lesões cutâneas em animais de experimentação (Mesocricetus auratus/hamster) por pelo menos seis meses de observação. As duas espécies no qual foram feitos os isolamentos (Tabela 2), a L. umbratilis e $L$. dendrophyla apresentavam sangue em decomposição e sangue fresco quando dissecadas.

Tabela 2. Origem e identificação de Leishmania sp. e cepas dereferência utilizadas neste estudo para identificação preliminar das amostras isoladasde flebotomíneos

\begin{tabular}{|c|c|c|c|}
\hline $\mathrm{NE}^{*}$ & Cepa de Referência & Espécie $^{* *}$ & Origem Geográfica \\
\hline 1245 & IHAR/CO/85/CL500 & L. colombiensis & Colômbia, Santander \\
\hline 1545 & MCEB/BR/84/M8408 & L. shawi & BR, PA, Serra dos Carajás \\
\hline 1367 & MCOE/BR/82/1367 & L. lainsoni & BR, RO, BR 364 \\
\hline $\mathrm{Z} 23$ & MHOM/BR/75/M4147 & L. guyanensis & BR, PA, Monte Dourado \\
\hline 562 & MHOM/PA/71/LS94 & L. panamensis & Panamá, Zona do Canal \\
\hline EZ01 & MCHO/BR/89/RO9627 & Endotrypanum sp. & $\mathrm{BR}, \mathrm{RO}$ \\
\hline EZ12 & MCHO/BR/88/M11602 & E. schaudinni & $\mathrm{BR}, \mathrm{PA}$ \\
\hline \multicolumn{4}{|c|}{ Isolados neste estudo (Origem Geográfica: BR, AM, Tabatinga) } \\
\hline NE* & Designação & $\mathrm{NE}^{*}$ & Designação \\
\hline 5789 & IUMB/BR/2010/IM5789 & 5803 & IUMB/BR/2010/IM5803 \\
\hline 5790 & IUMB/BR/2010/IM5790 & 5804 & IDEND/BR/2010/IM5804 \\
\hline 5791 & IUMB/BR/2010/IM5791 & 5805 & IUMB/BR/2010/IM5805 \\
\hline 5792 & IUMB/BR/2010/IM5792 & 5813 & IUMB/BR/2010/IM5813 \\
\hline 5794 & IDEND/BR/2010/IM5794 & 5905 & IUMB/BR/2011/IM5905 \\
\hline
\end{tabular}

NE: Número de Estoque. Legenda da Designação da Cepas: hospedeiros [M=Mammalia: HOM: Homo sapiens; CHO: Choloepus sp.; I: insecta; UMB: Lutzomya umbratilis; DEND: Lutzomyia dendrophyla; BR: país de origem (Brasil)/Ano de isolamento/Código original utilizado pelo INPA.

** identificação do estoque foi estabelecida através da análise de isoenzimas de acordo com o padrão isoenzimático e análise numérica (Momen, 1984; Cupolillo et al.,1994).

Os perfis bioquímicos foram comparados com diversas espécies do gênero Leishmania, mas apesar da semelhança com a $L$. (V.) colombiensis - que também não desenvolveu lesões em animais experimentais, apresentou no locus Malato Desidrogenase (MDH) apenas uma única banda (Rodriguez-Bonfante et al., 2003), ao contrário do observado em Endotrypanum para a maioria dos zimodemas (Franco et al., 1996).

\section{Conclusões}

O município de Tabatinga é uma região que pode ser considerada como área potencial para a circulação de leishmanias, devido à ocorrência de diversos vetores potenciais para a sua transmissão. O predomínio de L. umbratilis tanto em área silvestre como em peridomicilio, pode indicar a espécie como principal vetor envolvido na transmissão da LTA também nessa região, bem como a presença de várias outras espécies apontadas na transmissão de leishmaniose em área de peridomicilio serve de alerta para as autoridades da saúde.

\section{Agradecimentos}

Aos técnicos do Laboratório de Leishmaniose e Doença de Chagas, em especial a Artêmio Coelho, Francisco Lima e Lourival Maciel pela dedicação no trabalho de campo e a Coordenação de Aperfeiçoamento de Pessoal de Nível Superior (CAPES).

\section{Referências}

Aguiar, V.; Goncalves, G.M.S.; Farias, F.D. 2003. Distribuição dos casos de leishmaniose visceral humana em Pernambuco, Brasil em 2002. Revista da Sociedade Brasileira de Medicina Tropical, Brasília, 36 (2): 39-40, 2003 
Barrett, T.V.; Freitas, R.A.; Albuquerque, M.I.C.; Guerrero, J.H.C. 1996. Report on a collection of Lutzomyia sandflies (Diptera: Psychodidae) from the middle Solimões (Amazonas, Brazil). Memórias do Instituto Oswaldo Cruz, 91(1):27-35.

Castellón, E. G.; Arias, J.R.; Freitas, R. A.; Naiff, R. D. 1994. Os flebotomíneos da região Amazônica, estrada Manaus- Humaitá, estado do Amazonas, Brasil (Diptera: Psychodidae; Phlebotominae). Acta Amazônica, 24(1/2):91-102.

Chagas, A. P.; Soares, D. C.; Sousa, G. C. R.; V. R. B.; Rebelo, J. M. M.; Garcez, L. M. 2016. Aspectos ecológicos da fauna de flebotomíneos em focos de leishmaniose na Amazônia Oriental, Estado do Pará, Brasil. Revista Pan-Amazônica de Saude, 7(esp): 123-132.

Dias-Lima, A.; Bermúdez, E. C.; Medeiros, J. F.; Sherlock, I. 2002. Estratificação vertical da fauna de flebótomos (Diptera, Psychodidae) numa floresta primária de terra firme da Amazônia Central, Estado do Amazonas, Brasil. Caderno de Saúde Pública, Rio de Janeiro, 18(3):823-832.

IBGE, 2016. Estimativas da população residente no Brasil e Unidades da Federação com data de referência em $1^{\circ}$ de julho de 2016. Instituto Brasileiro de Geografia e Estatística. Acesso em 01/01/2017.

Ferro, C.; Morales, A. 1998. Flebótomos de Colômbia: estúdios realizados por el Laboratório de Entomologia 1966-1997. In: Toro, G.; Hernandez, C.A.; Raad, J. (E d.). Instituto Nacional de Salud 1917-1997: una historia un compromisso. Instituto Nacional de Salud, Bogotá, p. 21933.

Fé, N.A.; Freitas, R.A.; Barrett, T. B. 1998. Phlebotomine sand flies from São Gabriel da Cachoeira (State of Amazonas, Brazil) with a description of Lutzomyia (Psychodopygus) douradoi n.sp. (Diptera: Psychodidae). Memórias do Instituto Oswaldo Cruz, 93 (3): 331-336.

Figueira, L. P.; Zanotti, M.; Pinheiro, F. G.; Franco, A. M. R. 2008. Isoenzymatic characterization of human isolates of Leishmania sp (Kinetoplastida: Trypanosomatidae) from the municipalities of Rio Preto da Eva and Manaus, State of Amazonas. Revista da Sociedade Brasileira de Medina Tropical, 41(5): 512-514.

Franco, A. M. R.; Pereira, M. A. S.; Freitas, R. A. 2010. Diversidade da fauna de flebotomíneos em área de assentamento no município de Rio Preto da Eva, Estado do Amazonas. In: Castellon, E.G.B.; Ronchi-Teles, B.; Ale-Rocha, R. Org. (Org.). Entomologia na Amazônia Brasileira. v. 1. Editora INPA. Instituto Nacional de Pesquisas da Amazônia, Manaus, Amazonas, p.183-205.

Franco, A. M. R; Momen, H.; Naiff, R. D.; Moreira, C. F. S; Deane, M. P.; Grimaldi, JR. G. 1996. Enzyme polymorphism in Endotrypanum and numerical analysis of isoenzyme data. Parasitology, 113 (Pt 1): 39-48.

Freitas, R. A.; Naiff, R. D.; Barret, T. V. 2002. Species Diversity and Flagellate Infections in the Sand Fly Fauna near Porto Grande, State of Amapá, Brazil (Diptera: Psychodidae: Kinetoplastida: Trypanosomatidae). Memórias do Instituto Oswaldo Cruz, 97 (1): 53 59.

Forattini, O. P. 1973. Entomologia Médica. IV. Psychodidae. Leishmaniose. Bartonelose. Edgard Blucher, São Paulo. 658p.

Gomes, L. H. M.; Nery, L. C. R.; Pinheiro, F. G.; Freitas, R. A.; Franco, A. M. R. 2009. Fauna de flebotomíneos (Diptera: Psychodidae: Phlebotominae) em terra firme e planície fluvial na área de influência do gasoduto Coari - Manaus, Amazonas, Brasil. Acta Amazônica, 39 (1): 233-236.

Gomes, L. H. M.; Pinheiro, F. G.; Franco, A. M. R. 2010. Infecção e sazonalidade de vetores de Leishmaniose em área de treinamento militar no Amazonas. In: Castellon, E.G.B.; Ronchi-Teles, B.; Ale-Rocha, R. Org. (Org.). Entomologia na Amazônia Brasileira. v.1. Editora INPA. Instituto Nacional de Pesquisas da Amazônia, Manaus, Amazonas, p. 207-219.

Gontijo, C. M. F.; Melo, M. N. 2004. Leishmaniose Visceral No Brasil: Quadro Atual, Desafios e Perspectivas. Revista Brasileira de Epidemiologia, 7(3): 338-349,

Hendricks, L. D.; Wood, D. E.; Hadjuk, M. E. 1978. Haemoflagellates: commercially available liquid media for rapid cultivation. Parasitology. 76(3): 309- 316.

Killick-Kendrick, R. 1990. Phlebotomine vectors of the leishmaniases: a review. Medicine Veterinary Entomology, 4(1): 1-24.
Lainson, R.; Shaw, J. J.; Ready, P. D.; Miles, M. A., Póvoa, M. 1981. Leishmaniasis in Brazil. XVI. Isolation and identification of Leishmania species in North Pará State, with particular reference to Leishmania braziliensis guyanensis causative agent of "pian bois". Transactions of the Royal Society of Tropical Medicine and Hygiene, 75(4): 530-536.

Marcondes, C. B.; Santos-Neto, L. G.; Lozovei, A. L. 2001. Ecology the phlebotominae sandflies (Diptera: Psychodidae) in Brasilian Atlantic Florest. Revista da Sociedade Brasileira de Medicina Tropical, 34 (3): 255-260.

Neitzke, H. C.; Scodro, R. B. L.; Castro, K. R. R.; Sversutti, A. C. D.; Silveira, T. G. V.; Teodoro, U. 2008. Pesquisa de infecção natural de flebotomíneos por Leishmania, no Estado do Paraná. Revista da Sociedade Brasileira de Medicina Tropical, 41(1): 17-22.

Nery, L. C. R.; Freitas, R. A.; Franco, A. M. R. 2010. Fauna de flebotomíneos em fragmento florestal urbano no Amazonas. In: Castellón, E.G.B.; Ronchi-Teles, B.; Ale Rocha, R. (Org.). Entomologia na Amazônia Brasileira. v. 1. Editora INPA. Instituto Nacional de Pesquisas da Amazônia, Manaus, Amazonas, p. 247-261.

Nicolle, G. L. 1908. Culture du parasite du Bouton d'Orient. Comptes rendus de l'Académie des sciences, 146: 842-528.

Novy, F. G., MacNeal, W. J. 1904. On the cultivation of Trypanosoma brucei. Journal of Infectious Diseases, 1(1): 1-30.

Pessoa, F. A. C.; Medeiros, J. F.; Barret, T. V. 2007. Effects of timber harvest on phlebotomine sand flies (Diptera: Psychodidae) in a production forest: abundance of species on tree trunks and prevalence of trypanosomatids. Memórias do Instituto Oswaldo Cruz, 102(5): 593599.

Pinheiro, $\quad$ F. G.; Luz, $\quad$ S. L. B.; Franco, $\quad$ A. M. R. 2008. Infecção natural por tripanosomatídeos (Kinetoplastida: Trypanosomatidae) em Lutzomyia umbratilis (Diptera: Psychodidae) em áreas de leishmaniose tegumentar americana no Amazonas, Brasil. Acta Amazônica, 38(1):165-172.

Pirajá, G. V.; Lucheis, S. B. 2014. A vigilância epidemiológica de flebotomíneos no planejamento de ações de controle nas leishmanioses. Veterinária e Zootecnia, 21(4): 503-515.

Ready, P. D.; Arias, J. R.; Freitas, R. A. 1985. A pilot study to control Lutzomyia umbratilis (Diptera: Psychodidae), the major vector of Leishmania braziliensis guyanensis, in a peri-urban rainforest of Manaus, Amazonas state, Brazil. Memórias do Instituto Oswaldo Cruz, 80(1): 27-36.

Rodriguez-Bonfante, C.; Bonfante-Garrido, R.; Grimaldi, G. Jr.; Momen, H.; Cupolillo, E. 2003. Genotypically distinct Leishmania colombiensis isolates from Venezuela cause both cutaneous and visceral leishmaniasis in humans. Infection, Genetics and Evolution, 3(2): 119-24.

Ryan, L.; Silveira, F.T.; Lainson, R.; Shaw, J.J. 1987. Leishmaniasis in Brazil. XXIV. Natural flagellate infections of sandflies in Pará State, whit particular reference to the role of Psychodopygus wellcomei as the vector of Leishmania braziliensis braziliensis in the Serra dos Carajás. Transactions of the Royal Society of Tropical Medicine and Hygiene, 81(3): 353-59.

Santamaría, E.; Ponce, N.; Zipa, Y.; Ferro, C. 2006. Presencia en el peridomicilio de vectores infectados con Leishmania (Viannia) panamensis en dos focos endémicos em el occidente de Boyacá, piedemonte del valle del Magdalena medio, Colômbia. Biomédica 26(1): 82-94.

Sherlock, I. A. 2003. Importância Médico-Veterinária. In: Rangel, E. F.; Lainson, R. (Eds) Flebotomíneos do Brasil. Rio de Janeiro: Ed. Fiocruz, 2003, p.15-21.

Silva, D. F.; Freitas, R. A.; Franco, A. M. R. 2007. Diversidade e abundância de flebotomíneos do gênero Lutzomyia (Diptera: Psychodidae) em áreas de mata do nordeste de Manacapuru, AM. Neotropical Entomo$\log y, 36(1): 138-144$

Silveira, F. T.; Mülher, S. R.; Souza, A. A. A.; Lainson, R.; Gomes, C. M.; Laurent, M. D.; Corbett, C. E. P. 2008. Revisão sobre a patogenia da leishmaniose tegumentar americana na Amazônia, com ênfase à doença causada por Leishmania (V.) braziliensis e Leishmania (L.) amazonensis. Revista Paraense de Medicina, 22(1):9-20.

Shaw, J.J. 2003. New World leishmaniasis: the ecology of leishmaniasis and the diversity of leishmanial species in Central and South America. In: Farrell, J. (Ed.), World Class Parasites: Leishmania. Kluwer Academic Publishers, Boston; p. 11-31. 
Souza, A. A. A.; Silveira, F.T.; Lainson, R.; Barata, I.R.; Silva, M.G.S.; Lima, J.A.N.; Pinheiro, M.S.B.; Silva, F.M.M.; Vasconcelos, L.S.; Campos, M,B.; Ishikawa, E.A.Y. 2010. Fauna flebotomínica da Serra dos Carajás, Estado do Pará, Brasil, e sua possível implicação na transmissão da leishmaniose tegumentar americana. Revista Pan-Amazônica de Saúde, 1(1):45-51.

Vergara, D.; Carrillo, L. M.; Bejarano, E. E.; Vélez, I. D. 2008. Primer informe de Lutzomyia yuilli Young \& Porter, 1972 y Lutzomyia triramula (Fairchild \& Hertig 1952) (Diptera: Psychodidae) en el departamento de Caldas, Colombia. Biota Neotropical, 8(3): 251-253.

Young, D. G.; Duncan, M. A. 1994. Guide of the identification and geographic distribution of Lutzomyia sand flies in Mexico, the West Indies, Central and South America (Diptera: Psychodidae). Memoirs of the American Entomological Institute, 54:1-881. 